\title{
Radii problems for normalized q-Bessel and Wright functions
}

\author{
Evrim Toklu \\ Department of Mathematics, \\ Faculty of Education, \\ A ̆̆rı İbrahim Çeçen University, \\ Ağrl, Turkey \\ email: etoklu@agri.edu.tr
}

\author{
İbrahim Aktaş \\ Department of Mathematics, \\ Kamil Özdă̆ Science Faculty, \\ Karamanoğlu Mehmetbey University, \\ 70100, Karaman, Turkey \\ email: aktasibrahim38@gmail.com
}

\author{
Halit Orhan \\ Department of Mathematics, \\ Faculty of Science, \\ Atatürk University, Erzurum, Turkey \\ email: orhanhalit607@gmail.com
}

\begin{abstract}
In this investigation, our main objective is to ascertain the radii of k-uniform convexity of order $\alpha$ and the radii of strong starlikeness of the some normalized q-Bessel and Wright functions. In making this investigation we deal with the normalized Wright functions for three different kinds of normalization and six different normalized forms of qBessel functions. The key tools in the proof of our main results are the Mittag-Leffler expansion for Wright and q-Bessel functions and properties of real zeros of these functions and their derivatives. We also have shown that the obtained radii are the smallest positive roots of some functional equations.
\end{abstract}

2010 Mathematics Subject Classification: 30C45, 30C15, 33C10

Key words and phrases: k-uniform convex functions; radius of k-uniform convexity of order $\alpha$; Mittag-Leffler expansions; Wright and q-Bessel functions; strong starlikeness 


\section{Introduction}

Special functions have an indispensable role in many branches of mathematics and applied mathematics. Thus, it is important to examine their properties in many aspects. In the recent years, there has been a vivid interest on some special functions from the point of view of geometric function theory. For more details we refer to the papers $[1,2,3,4,6,7,8,9,10,11,12,13,14$, $15,16,17,18]$ and references therein. However, the origins of these studies can be traced to Brown [20], to Kreyszig and Todd [22], and to Wilf [24]. These studies initiated investigation on the univalence of Bessel functions and determining the radius of starlikeness for different kinds of normalization. In other words, their results have a very important place on account of the fact that they have paved the way for obtaining other geometric properties of Bessel function such as univalence, starlikeness, convexity and so forth. Recently, in 2014, Baricz et al. [11], by considering a much simpler approach, succeeded to determine the radius of starlikeness of the normalized Bessel functions. In the same year, Baricz and Szász [15] obtained the radius of convexity of the normalized Bessel functions. We see in their proofs that some properties of the zeros of Bessel functions and the Mittag-Leffler expansions for Bessel function of the first kind play a crucial role in determining the radii of starlikeness and convexity of Bessel functions of the first kind. It is worth to mention that some geometric properties of other special functions involving Bessel function of first kind were investigated extensively by several authors. For instance, in 2017, Deniz and Szász [21] studied on determining the radius of uniform convexity of the normalized Bessel functions. And also, very recently, Bohara and Ravichandran in [19] determined, by using the method of Baricz et al. $[11,15,16,21]$, the radius of strong starlikeness and $k$-uniform convexity of order $\alpha$ of the normalized Bessel functions.

Inspired by the above mentioned results and considering the approach of Baricz et al. in this paper, we investigate the radius of strong starlikeness and $k$-uniform convexity of order $\alpha$ of the normalized Wright and $q-B e s s e l$ functions.

This paper is organized as follows: The rest of this section contains some basic definitions needed for the proof of our main results. Section 2 is divided into three subsections: The first subsection is devoted to the radii of $k$-uniform convexity of order $\alpha$ of normalized Wright functions. The second subsection contains the study of the radii of $k$-uniform convexity of order $\alpha$ of normalized $q-$ Bessel functions. The third subsection is dedicated to the radius of strong starlikeness of normalized Wright and q-Bessel functions. 
Before starting to present our main results we would like to call attention to some basic concepts, which are used by us for building our main results. For $r>0$ we denote by $\mathbb{D}_{r}=\{z \in \mathbb{C}:|z|<r\}$ the open disk with radius $r$ centered at the origin. Let $f: \mathbb{D}_{r} \rightarrow \mathbb{C}$ be the function defined by

$$
f(z)=z+\sum_{n \geq 2} a_{n} z^{n},
$$

here $r$ is less or equal than the radius of convergence of the above power series. Let $\mathcal{A}$ be the class of analytic functions of the form (1), that is, normalized by the conditions $\mathrm{f}(0)=\mathrm{f}^{\prime}(0)-1=0$. Let $\mathcal{S}$ denote the subclass of $\mathcal{A}$ consisting of univalent functions.

In this paper, for $k \geq 0$ and $0 \leq \alpha<1$ we study on more general class $\mathcal{U C V}(k, \alpha)$ of $k$-uniformly convex functions of order $\alpha$. A function $\mathrm{f} \in \mathcal{A}$ is said to be in the class $\mathcal{U C V}(k, \alpha)$ if

$$
\operatorname{Re}\left(1+\frac{z f^{\prime \prime}(z)}{f^{\prime}(z)}\right)>k\left|\frac{z f^{\prime \prime}(z)}{f^{\prime}(z)}\right|+\alpha \quad(z \in \mathbb{D}) .
$$

The real number

$$
r_{k, \alpha}^{u \mathfrak{c}}(f)=\sup \left\{r>0\left|\operatorname{Re}\left(\frac{z f^{\prime}(z)}{f(z)}\right)>k\right| \frac{z f^{\prime \prime}(z)}{f^{\prime}(z)} \mid+\alpha \text { for all } z \in \mathbb{D}_{r}\right\}
$$

is called the radius of $k$-uniform convexity of order $\alpha$ of the function $f$.

Finally, let us take a look at the next lemma which is very useful in building our main results. It is worth to mention that the following lemma was proven by Deniz and Szász [21].

Lemma 1 (see [21]) If $\mathrm{a}>\mathrm{b}>\mathrm{r} \geq|z|$, and $\lambda \in[0,1]$, then

$$
\left|\frac{z}{b-z}-\lambda \frac{z}{a-z}\right| \leq \frac{r}{b-r}-\lambda \frac{r}{a-r} \text {. }
$$

The followings can be obtained as a natural consequence of this inequality:

$$
\operatorname{Re}\left(\frac{z}{b-z}-\lambda \frac{z}{a-z}\right) \leq \frac{r}{b-r}-\lambda \frac{r}{a-r}
$$

and

$$
\operatorname{Re}\left(\frac{z}{b-z}\right) \leq\left|\frac{z}{b-z}\right| \leq \frac{r}{b-r}
$$

We are now in a position to present our main results. 


\section{Main results}

\subsection{The radii of k-uniform convexity of order $\alpha$ of normalized Wright functions}

In this subsection, we will focus on the function

$$
\phi(\rho, \beta, z)=\sum_{n \geq 0} \frac{z^{n}}{n ! \Gamma(n \rho+\beta)} \quad(\rho>-1 \quad z, \beta \in \mathbb{C})
$$

named after the British mathematician E.M. Wright. It is well known that this function was introduced by him for the first time in the case $\rho>0$ in connection with his investigations on the asymptotic theory of partitions [26].

From [17, Lem. 1] we know that under the conditions $\rho>0$ and $\beta>0$, the function $z \mapsto \lambda_{\rho, \beta}(z)=\phi\left(\rho, \beta,-z^{2}\right)$ has infinitely many zeros which are all real. Thus, in light of the Hadamard factorization theorem, the infinite product representation of the function $\lambda_{\rho, \beta}(z)$ can be written as

$$
\Gamma(\beta) \lambda_{\rho, \beta}(z)=\prod_{n \geq 1}\left(1-\frac{z^{2}}{\lambda_{\rho, \beta, n}^{2}}\right)
$$

where $\lambda_{\rho, \beta, n}$ is the $n$th positive zero of the function $\lambda_{\rho, \beta}(z)$ (or the positive real zeros of the function $\Psi_{\rho, \beta}$ ). Moreover, let $\zeta_{\rho, \beta, n}^{\prime}$ denote the $n$th positive zero of $\Psi_{\rho, \beta}^{\prime}$, where $\Psi_{\rho, \beta}(z)=z^{\beta} \lambda_{\rho, \beta}(z)$, then the zeros satisfy the chain of inequalities

$$
\zeta_{\rho, \beta, 1}^{\prime}<\zeta_{\rho, \beta, 1}=\lambda_{\rho, \beta, 1}<\zeta_{\rho, \beta, 2}^{\prime}<\zeta_{\rho, \beta, 2}=\lambda_{\rho, \beta, 2}<\ldots
$$

One can easily see that the function $z \mapsto \phi\left(\rho, \beta,-z^{2}\right)$ do not belong to $\mathcal{A}$, and thus first we perform some natural normalizations. We define three functions originating from $\phi(\rho, \beta,$.$) :$

$$
\begin{aligned}
f_{\rho, \beta}(z) & =\left(z^{\beta} \Gamma(\beta) \phi\left(\rho, \beta,-z^{2}\right)\right)^{\frac{1}{\beta}}, \\
g_{\rho, \beta}(z) & =z \Gamma(\beta) \phi\left(\rho, \beta,-z^{2}\right), \\
h_{\rho, \beta}(z) & =z \Gamma(\beta) \phi(\rho, \beta,-z) .
\end{aligned}
$$

Clearly, these functions are contained in the class $\mathcal{A}$.

Now, we would like to present our results regarding the $k$-uniform convexity of order $\alpha$ of the functions $f_{\rho, \beta}, g_{\rho, \beta}$ and $h_{\rho, \beta}$. 
Theorem 1 Let $\beta, \rho>0, \alpha \in[0,1)$ and $k \geq 0$. Then, the following statements are valid:

a. The radius of $k$-uniform convexity of order $\alpha$ of the function $f_{\rho, \beta}$ is the real number $r_{k, \alpha}^{u c}\left(f_{\rho, \beta}\right)$ which is the smallest positive root of the equation

$$
(1+k) r \frac{\Psi_{\rho, \beta}^{\prime \prime}(r)}{\Psi_{\rho, \beta}^{\prime}}+\left(\frac{1}{\beta}-1\right)(1+k) r \frac{\Psi_{\rho, \beta}^{\prime}(r)}{\Psi_{\rho, \beta}(r)}+1-\alpha=0
$$

in the interval $\left(0, \zeta_{\rho, \beta, 1}^{\prime}\right)$, where $\Psi_{\rho, \beta}(z)=z^{\beta} \lambda_{\rho, \beta}(z)$ and $\zeta_{\rho, \beta, 1}^{\prime}$ stands for the smallest positive zero of the function $\Psi_{\rho, \beta}^{\prime}(z)$.

b. The radius of $\mathrm{k}$-uniform convexity of order $\alpha$ of the function $\mathrm{g}_{\rho, \beta}$ is the real number $\mathrm{r}_{k, \alpha}^{\mathrm{uc}}\left(\mathrm{g}_{\rho, \beta}\right)$ which is the smallest positive root of the equation

$$
(1+k) r \frac{g_{\rho, \beta}^{\prime \prime}(r)}{g_{\rho, \beta}^{\prime}(r)}+1-\alpha=0
$$

in the interval $\left(0, \vartheta_{\rho, \beta, 1}\right)$, where $\vartheta_{\rho, \beta, 1}$ stands for the smallest positive zero of the function $\mathrm{g}_{\rho, \beta}^{\prime}(z)$.

c. The radius of $k$-uniform convexity of order $\alpha$ of the function $h_{\rho, \beta}$ is the real number $\mathrm{r}_{k, \alpha}^{\mathrm{uc}}\left(\mathrm{h}_{\rho, \beta}\right)$ which is the smallest positive root of the equation

$$
(1+k) r \frac{h_{\rho, \beta}^{\prime \prime}(r)}{h_{\rho, \beta}^{\prime}(r)}+1-\alpha=0
$$

in the interval $\left(0, \tau_{\rho, \beta, 1}\right)$, where $\tau_{\rho, \beta, 1}$ stands for the smallest positive zero of the function $\mathrm{h}_{\rho, \beta}^{\prime}(z)$

\section{Proof.}

a. We note that

$$
1+\frac{z f_{\rho, \beta}^{\prime \prime}(z)}{f_{\rho, \beta}^{\prime}(z)}=1+\frac{z \Psi_{\rho, \beta}^{\prime \prime}(z)}{\Psi_{\rho, \beta}^{\prime}(z)}+\left(\frac{1}{\beta}-1\right) \frac{z \Psi_{\rho, \beta}^{\prime}(z)}{\Psi_{\rho, \beta}(z)} .
$$

Using the following infinite product representations of $\Psi_{\rho, \beta}$ and $\Psi_{\rho, \beta}^{\prime}[17$, Theorem 5] given by

$$
\Gamma(\beta) \Psi_{\rho, \beta}(z)=z^{\beta} \prod_{n \geq 1}\left(1-\frac{z^{2}}{\zeta_{\rho, \beta, n}^{2}}\right), \Gamma(\beta) \Psi_{\rho, \beta}^{\prime}(z)=z^{\beta-1} \prod_{n \geq 1}\left(1-\frac{z^{2}}{\zeta_{\rho, \beta, n}^{\prime 2}}\right),
$$


where $\zeta_{\rho, \beta, n}$ and $\zeta_{\rho, \beta, n}^{\prime}$ denote the $n$th positive roots of $\Psi_{\rho, \beta}$ and $\Psi_{\rho, \beta}^{\prime}$, respectively, we have

$$
\frac{z \Psi_{\rho, \beta}^{\prime}(z)}{\Psi_{\rho, \beta}(z)}=\beta-\sum_{n \geq 1} \frac{2 z^{2}}{\zeta_{\rho, \beta, n}^{2}-z^{2}}, \quad \frac{z \Psi_{\rho, \beta}^{\prime \prime}(z)}{\Psi_{\rho, \beta}^{\prime}(z)}=\beta-1-\sum_{n \geq 1} \frac{2 z^{2}}{\zeta_{\rho, \beta, n}^{\prime 2}-z^{2}} .
$$

Thus we arrive at

$$
1+\frac{z f_{\rho, \beta}^{\prime \prime}(z)}{f_{\rho, \beta}^{\prime}(z)}=1-\left(\frac{1}{\beta}-1\right) \sum_{n \geq 1} \frac{2 z^{2}}{\zeta_{\rho, \beta, n}^{2}-z^{2}}-\sum_{n \geq 1} \frac{2 z^{2}}{\zeta_{\rho, \beta, n}^{\prime 2}-z^{2}} .
$$

In order to prove the theorem we consider two cases $\beta \in(0,1]$ and $\beta>1$ separately.

Case $1 \beta \in(0,1]$.

Then $\lambda=\frac{1}{\beta}-1>0$. By making use of inequality (4) stated in Lemma 1 we conclude that the following inequality

$$
\frac{|z|^{2}}{\zeta_{\rho, \beta, n}^{2}-|z|^{2}} \geq \operatorname{Re}\left(\frac{z^{2}}{\zeta_{\rho, \beta, n}^{2}-z^{2}}\right)
$$

holds true for every $\rho>0, \beta>0, n \in \mathbb{N}$ and $|z|<\zeta_{\rho, \beta, n}$. With the help of (4), we get

$$
\begin{aligned}
\operatorname{Re}\left(1+\frac{z f_{\rho, \beta}^{\prime \prime}(z)}{f_{\rho, \beta}^{\prime}(z)}\right) & \geq 1-\left(\frac{1}{\beta}-1\right) \sum_{n \geq 1} \frac{2 r^{2}}{\zeta_{\rho, \beta, n}^{2}-r^{2}}-\sum_{n \geq 1} \frac{2 r^{2}}{\zeta_{\rho, \beta, n}^{\prime 2}-r^{2}} \\
& =1+\frac{r f_{\rho, \beta}^{\prime \prime}(r)}{f_{\rho, \beta}^{\prime}(r)},
\end{aligned}
$$

where $|z|=r$ and $z \in \mathbb{D}_{\zeta_{\rho, \beta, 1}^{\prime}}$.

Moreover, by using triangle inequality $\left|z_{1}+z_{2}\right| \leq\left|z_{1}\right|+\left|z_{2}\right|$ together with the fact that $\frac{1}{\beta}-1>0$, we get 


$$
\begin{aligned}
\left|\frac{z f_{\rho, \beta}^{\prime \prime}(z)}{f_{\rho, \beta}^{\prime}(z)}\right| & =\left|\sum_{n \geq 1} \frac{2 z^{2}}{\zeta_{\rho, \beta, n}^{\prime 2}-z^{2}}+\left(\frac{1}{\beta}-1\right) \sum_{n \geq 1} \frac{2 z^{2}}{\zeta_{\rho, \beta, n}^{2}-z^{2}}\right| \\
& \leq \sum_{n \geq 1}\left|\left(\frac{2 z^{2}}{\zeta_{\rho, \beta, n}^{\prime 2}-z^{2}}+\left(\frac{1}{\beta}-1\right) \frac{2 z^{2}}{\zeta_{\rho, \beta, n}^{2}-z^{2}}\right)\right| \\
& \leq \sum_{n \geq 1}\left(\frac{2 r^{2}}{\zeta_{\rho, \beta, n}^{\prime 2}-r^{2}}+\left(\frac{1}{\beta}-1\right) \frac{2 r^{2}}{\zeta_{\rho, \beta, n}^{2}-r^{2}}\right) \\
& =-\frac{r f_{\rho, \beta}^{\prime \prime}(r)}{f_{\rho, \beta}^{\prime}(r)} .
\end{aligned}
$$

From (5) and (6), we obtain

$$
\begin{gathered}
\operatorname{Re}\left(1+\frac{z f_{\rho, \beta}^{\prime \prime}(z)}{f_{\rho, \beta}^{\prime}(z)}\right)-k\left|\frac{z f_{\rho, \beta}^{\prime \prime}(z)}{f_{\rho, \beta}^{\prime}(z)}\right|-\alpha \geq 1+(1+k) r \frac{f_{\rho, \beta}^{\prime \prime}(r)}{f_{\rho, \beta}^{\prime}(r)}-\alpha, \\
|z| \leq r<\zeta_{\rho, \beta, 1}^{\prime} .
\end{gathered}
$$

Case $2 \beta>1$. Then, we show that the same inequality is valid in this case also. In this case, taking into consideration the inequality (3) stated in 1 we get

$$
\begin{aligned}
\operatorname{Re}\left(1+\frac{z f_{\rho, \beta}^{\prime \prime}(z)}{f_{\rho, \beta}^{\prime}(z)}\right) & \geq 1-\left(\frac{1}{\beta}-1\right) \sum_{n \geq 1} \frac{2 r^{2}}{\zeta_{\rho, \beta, n}^{2}-r^{2}}-\sum_{n \geq 1} \frac{2 r^{2}}{\zeta_{\rho, \beta, n}^{\prime 2}-r^{2}} \\
& =1+\frac{r f_{\rho, \beta}^{\prime \prime}(r)}{f_{\rho, \beta}^{\prime}(r)} .
\end{aligned}
$$

Also, with the aid of (2) stated in the same lemma, we have

$$
\begin{aligned}
\left|\frac{z f_{\rho, \beta}^{\prime \prime}(z)}{f_{\rho, \beta}^{\prime}(z)}\right| & =\left|\sum_{n \geq 1} \frac{2 z^{2}}{\zeta_{\rho, \beta, n}^{\prime 2}-z^{2}}-\left(1-\frac{1}{\beta}\right) \sum_{n \geq 1} \frac{2 z^{2}}{\zeta_{\rho, \beta, n}^{2}-z^{2}}\right| \\
& \leq \sum_{n \geq 1}\left|\left(\frac{2 z^{2}}{\zeta_{\rho, \beta, n}^{\prime 2}-z^{2}}-\left(1-\frac{1}{\beta}\right) \frac{2 z^{2}}{\zeta_{\rho, \beta, n}^{2}-z^{2}}\right)\right| \\
& \leq \sum_{n \geq 1}\left(\frac{2 r^{2}}{\zeta_{\rho, \beta, n}^{\prime 2}-r^{2}}-\left(1-\frac{1}{\beta}\right) \frac{2 r^{2}}{\zeta_{\rho, \beta, n}^{2}-r^{2}}\right) \\
& =-\frac{r f_{\rho, \beta}^{\prime \prime}(r)}{f_{\rho, \beta}^{\prime}(r)} .
\end{aligned}
$$


From (8) and (9), we deduce

$$
\begin{gathered}
\operatorname{Re}\left(1+\frac{z f_{\rho, \beta}^{\prime \prime}(z)}{f_{\rho, \beta}^{\prime}(z)}\right)-k\left|\frac{z f_{\rho, \beta}^{\prime \prime}(z)}{f_{\rho, \beta}^{\prime}(z)}\right|-\alpha \geq 1+(1+k) r \frac{f_{\rho, \beta}^{\prime \prime}(r)}{f_{\rho, \beta}^{\prime}(r)}-\alpha, \\
|z| \leq r<\zeta_{\rho, \beta, 1}^{\prime} .
\end{gathered}
$$

Due to the minimum principle for harmonic functions, equality holds if and only if $z=r$. Now, the above deduced inequalities imply for $r \in\left(0, \zeta_{\rho, \beta, 1}^{\prime}\right)$

$$
\inf _{z \in \mathbb{D}_{r}}\left\{\operatorname{Re}\left(1+\frac{z f_{\rho, \beta}^{\prime \prime}(z)}{f_{\rho, \beta}^{\prime}(z)}\right)-k\left|\frac{z f_{\rho, \beta}^{\prime \prime}(z)}{f_{\rho, \beta}^{\prime}(z)}\right|-\alpha\right\}=1-\alpha+(1+k) r \frac{f_{\rho, \beta}^{\prime \prime}(r)}{f_{\rho, \beta}^{\prime}(r)} .
$$

On the other hand, the function $u_{\rho, \beta}:\left(0, \zeta_{\rho, \beta, 1}^{\prime}\right) \rightarrow \mathbb{R}$ is defined by

$$
\begin{aligned}
u_{\rho, \beta}(r) & =1-\alpha+(1+k) r \frac{f_{\rho, \beta}^{\prime \prime}(r)}{f_{\rho, \beta}^{\prime}(r)} \\
& =1-\alpha+(1+k)\left(\sum_{n \geq 1} \frac{2 r^{2}}{\zeta_{\rho, \beta, n}^{\prime 2}-r^{2}}-\left(1-\frac{1}{\beta}\right) \sum_{n \geq 1} \frac{2 r^{2}}{\zeta_{\rho, \beta, n}^{2}-r^{2}}\right) .
\end{aligned}
$$

Then,

$$
\begin{aligned}
u_{\rho, \beta}^{\prime}(r)= & -4(1+k)\left(\frac{1}{\beta}-1\right) \sum_{n \geq 1} \frac{\zeta_{\rho, \beta, n}^{2} r}{\left(\zeta_{\rho, \beta, n}^{2}-r^{2}\right)^{2}} \\
& -4(k+1) \sum_{n \geq 1} \frac{\zeta_{\rho, \beta, n}^{\prime 2} r}{\left(\zeta_{\rho, \beta, n}^{\prime 2}-r^{2}\right)^{2}}<0
\end{aligned}
$$

for all $\beta \in(0,1]$ and $z \in \mathbb{D}_{\zeta_{\rho, \beta, 1}^{\prime}}$. Moreover, we consider that if $\beta>1$, then $0<1-1 / \beta<1$ and taking into consideration the inequality $\zeta_{\rho, \beta, n}^{2}\left(\zeta_{\rho, \beta, n}^{\prime 2}-r^{2}\right)^{2}<\zeta_{\rho, \beta, n}^{\prime 2}\left(\zeta_{\rho, \beta, n}^{2}-r^{2}\right)^{2}$ for $r<\zeta_{\rho, \beta, 1}^{\prime}$, we get

$$
\begin{aligned}
u_{\rho, \beta}^{\prime}(r) & =-4(1+k)\left(\frac{1}{\beta}-1\right) \sum_{n \geq 1} \frac{\zeta_{\rho, \beta, n}^{2} r}{\left(\zeta_{\rho, \beta, n}^{2}-r^{2}\right)^{2}}-4(k+1) \sum_{n \geq 1} \frac{\zeta_{\rho, \beta, n}^{\prime 2} r}{\left(\zeta_{\rho, \beta, n}^{\prime 2}-r^{2}\right)^{2}} \\
& <4(1+k)\left(\sum_{n \geq 1} \frac{\zeta_{\rho, \beta, n}^{2} r}{\left(\zeta_{\rho, \beta, n}^{2}-r^{2}\right)^{2}}-\sum_{n \geq 1} \frac{\zeta_{\rho, \beta, n}^{\prime 2} r}{\left(\zeta_{\rho, \beta, n}^{\prime 2}-r^{2}\right)^{2}}\right)<0 .
\end{aligned}
$$


Consequently, $\mathfrak{u}_{\rho, \beta}$ is strictly decreasing function of $r$ for all $\beta>0$. Also,

$$
\lim _{r \searrow 0} u_{\rho, \beta}(r)=1-\alpha \text { and } \lim _{r / \zeta_{\rho, \beta, 1}^{\prime}} u_{\rho, \beta}(r)=-\infty
$$

This means that

$$
\operatorname{Re}\left(1+\frac{z f_{\rho, \beta}^{\prime \prime}(z)}{f_{\rho, \beta}^{\prime}(z)}\right)-k\left|\frac{z f_{\rho, \beta}^{\prime \prime}(z)}{f_{\rho, \beta}^{\prime}(z)}\right|-\alpha>0
$$

for all $z \in \mathbb{D}_{r_{k, \alpha}^{u c}\left(f_{\rho, \beta}\right)}$ where $r_{k, \alpha}^{u c}\left(f_{\rho, \beta}\right)$ is the unique root of the equation

$$
1-\alpha+(1+k) r \frac{f_{\rho, \beta}^{\prime \prime}(r)}{f_{\rho, \beta}^{\prime}(r)}=0
$$

or

$$
(1+k) r \frac{\Psi_{\rho, \beta}^{\prime \prime}(r)}{\Psi_{\rho, \beta}^{\prime}}+\left(\frac{1}{\beta}-1\right)(1+k) r \frac{\Psi_{\rho, \beta}^{\prime}(r)}{\Psi_{\rho, \beta}(r)}+1-\alpha=0
$$

in $\left(0, \zeta_{\rho, \beta, 1}^{\prime}\right)$.

b. Let $\vartheta_{\rho, \beta, n}$ be the $n$th positive zero of the function $g_{\rho, \beta}^{\prime}(z)$. In view of the Hadamard theorem we get the Weierstrassian canonical representation (see [17])

$$
g_{\rho, \beta}^{\prime}(z)=\prod_{n \geq 1}\left(1-\frac{z^{2}}{\vartheta_{\rho, \beta, n}^{2}}\right) .
$$

Logarithmic derivation of both sides yields

$$
1+\frac{z g_{\rho, \beta}^{\prime \prime}(z)}{g_{\rho, \beta}^{\prime}(z)}=1-\sum_{n \geq 1} \frac{2 z^{2}}{\vartheta_{\rho, \beta, n}^{2}-z^{2}} .
$$

Application of the inequality (4) implies that

$$
\operatorname{Re}\left(1+\frac{z g_{\rho, \beta}^{\prime \prime}(z)}{g_{\rho, \beta}^{\prime}(z)}\right) \geq 1-\sum_{n \geq 1} \frac{2 r^{2}}{\vartheta_{\rho, \beta, n}^{2}-r^{2}},
$$

where $|z|=r$. Moreover,

$$
\begin{aligned}
\left|\frac{z g_{\rho, \beta}^{\prime \prime}(z)}{g_{\rho, \beta}^{\prime}(z)}\right| & =\left|\sum_{n \geq 1} \frac{2 z^{2}}{\vartheta_{\rho, \beta, n}^{2}-z^{2}}\right| \leq \sum_{n \geq 1}\left|\frac{2 z^{2}}{\vartheta_{\rho, \beta, n}^{2}-z^{2}}\right| \leq \sum_{n \geq 1} \frac{2 r^{2}}{\vartheta_{\rho, \beta, n}^{2}-r^{2}} \\
& =-\frac{r g_{\rho, \beta}^{\prime \prime}(r)}{g_{\rho, \beta}^{\prime}(r)}, \quad|z| \leq r<\vartheta_{\rho, \beta, 1} .
\end{aligned}
$$


Taking into considering the inequalities (11) and (12) we arrive at $\operatorname{Re}\left(1+\frac{z g_{\rho, \beta}^{\prime \prime}(z)}{g_{\rho, \beta}^{\prime}(z)}\right)-k\left|\frac{z g_{\rho, \beta}^{\prime \prime}(z)}{g_{\rho, \beta}^{\prime}(z)}\right|-\alpha \geq 1-\alpha+(1+k) r \frac{g_{\rho, \beta}^{\prime \prime}(r)}{g_{\rho, \beta}^{\prime}(r)}|z|<r<\vartheta_{\rho, \beta, 1}$.

In light of the minimum principle for harmonic functions, equality holds if and only if $z=r$. Thus, for $r \in\left(0, \vartheta_{\rho, \beta, 1}\right)$ we get

$\inf _{|z|<r}\left\{\operatorname{Re}\left(1+\frac{z g_{\rho, \beta}^{\prime \prime}(z)}{g_{\rho, \beta}^{\prime}(z)}\right)-k\left|\frac{z g_{\rho, \beta}^{\prime \prime}(z)}{g_{\rho, \beta}^{\prime}(z)}\right|-\alpha\right\}=1-\alpha+(1+k) r \frac{g_{\rho, \beta}^{\prime \prime}(r)}{g_{\rho, \beta}^{\prime}(r)}$.

The function $w_{\rho, \beta}:\left(0, \vartheta_{\rho, \beta, 1}\right) \rightarrow \mathbb{R}$, defined by

$$
w_{\rho, \beta}(\mathrm{r})=1-\alpha+(1+\mathrm{k}) \mathrm{r} \frac{\mathrm{g}_{\rho, \beta}^{\prime \prime}(\mathrm{r})}{g_{\rho, \beta}^{\prime}(\mathrm{r})},
$$

is strictly decreasing and

$$
\lim _{r \searrow 0} w_{\rho, \beta}(r)=1-\alpha>0, \quad \lim _{r / \vartheta_{\rho, \beta, 1}} w_{\rho, \beta}(r)=-\infty
$$

Consequently,

$$
\operatorname{Re}\left(1+\frac{z g_{\rho, \beta}^{\prime \prime}(z)}{g_{\rho, \beta}^{\prime}(z)}\right)-k\left|\frac{z g_{\rho, \beta}^{\prime \prime}(z)}{g_{\rho, \beta}^{\prime}(z)}\right|-\alpha>0
$$

for all $\mathbb{D}_{r_{k, \alpha}^{u c}\left(g_{\rho, \beta}\right)}$ where $r_{k, \alpha}^{u c}\left(g_{\rho, \beta}\right)$ is the unique root of the equation

$$
1-\alpha+(1+k) r \frac{g_{\rho, \beta}^{\prime \prime}(r)}{g_{\rho, \beta}^{\prime}(r)}=0
$$

in $\left(0, \vartheta_{\rho, \beta, 1}\right)$.

c. Let $\tau_{\rho, \beta, n}$ denote the $n$th positive zero of the function $h_{\rho, \beta}^{\prime}$. By using again the fact that the zeros of the Wright function $\lambda_{\rho, \beta}$ are all real and in view of the Hadamard theorem we obtain

$$
h_{\rho, \beta}^{\prime}(z)=\prod_{n \geq 1}\left(1-\frac{z}{\tau_{\rho, \beta, n}}\right),
$$

which implies that

$$
1+\frac{z h_{\rho, \beta}^{\prime \prime}(z)}{h_{\rho, \beta}^{\prime}(z)}=1-\sum_{n \geq 1} \frac{z}{\tau_{\rho, \beta, n}-z} .
$$


By using again the inequaliy (4) we get

$$
\operatorname{Re}\left(1+\frac{z h_{\rho, \beta}^{\prime \prime}(z)}{h_{\rho, \beta}^{\prime}(z)}\right) \geq 1-\sum_{n \geq 1} \frac{r}{\tau_{\rho, \beta, n}-r}=1+r \frac{h_{\rho, \beta}^{\prime \prime}(r)}{h_{\rho, \beta}^{\prime}(r)}
$$

Also,

$$
\left|\frac{z h_{\rho, \beta}^{\prime \prime}(z)}{h_{\rho, \beta}^{\prime}(z)}\right|=\left|-\sum_{n \geq 1} \frac{z}{\tau_{\rho, \beta, n}-z}\right| \leq \sum_{n \geq 1} \frac{r}{\tau_{\rho, \beta, n}-r}=-r \frac{h_{\rho, \beta}^{\prime \prime}(r)}{h_{\rho, \beta}^{\prime}(r)} .
$$

Considering the inequalities (13) and (14) we have

$$
\operatorname{Re}\left(1+\frac{z g_{\rho, \beta}^{\prime \prime}(z)}{g_{\rho, \beta}^{\prime}(z)}\right)-k\left|\frac{z g_{\rho, \beta}^{\prime \prime}(z)}{g_{\rho, \beta}^{\prime}(z)}\right|-\alpha \geq 1-\alpha+(1+k) r \frac{g_{\rho, \beta}^{\prime \prime}(r)}{g_{\rho, \beta}^{\prime}(r)} .
$$

In view of the minimum principle for harmonic functions, equality holds if and only if $z=r$. Thus, for $r \in\left(0, \tau_{\rho, \beta, 1}\right)$ we have

$$
\inf _{|z|<r}\left\{\operatorname{Re}\left(1+\frac{z h_{\rho, \beta}^{\prime \prime}(z)}{h_{\rho, \beta}^{\prime}(z)}\right)-k\left|\frac{z h_{\rho, \beta}^{\prime \prime}(z)}{h_{\rho, \beta}^{\prime}(z)}\right|-\alpha\right\}=1-\alpha+(1+k) r \frac{h_{\rho, \beta}^{\prime \prime}(r)}{h_{\rho, \beta}^{\prime}(r)} .
$$

Now define the function $\varphi_{\rho, \beta}:\left(0, \vartheta_{\rho, \beta, 1}\right) \rightarrow \mathbb{R}$, as

$$
\varphi_{\rho, \beta}(r)=1-\alpha+(1+k) r \frac{h_{\rho, \beta}^{\prime \prime}(r)}{h_{\rho, \beta}^{\prime}(r)}
$$

is strictly decreasing and

$$
\lim _{r \searrow 0} \varphi_{\rho, \beta}(r)=1-\alpha>0, \quad \lim _{r / \vartheta_{\rho, \beta, 1}} \varphi_{\rho, \beta}(r)=-\infty .
$$

Consequently,

$$
\operatorname{Re}\left(1+\frac{z h_{\rho, \beta}^{\prime \prime}(z)}{h_{\rho, \beta}^{\prime}(z)}\right)-k\left|\frac{z h_{\rho, \beta}^{\prime \prime}(z)}{h_{\rho, \beta}^{\prime}(z)}\right|-\alpha>0
$$

for all $\mathbb{D}_{r_{k, \alpha}^{u c}\left(h_{\rho, \beta}\right)}$ where $r_{k, \alpha}^{u c}\left(h_{\rho, \beta}\right)$ is the unique root of equation

$$
1-\alpha+(1+k) r \frac{h_{\rho, \beta}^{\prime \prime}(r)}{h_{\rho, \beta}^{\prime}(r)}=0
$$

in $\left(0, \tau_{\rho, \beta, 1}\right)$. This completes the proof.

Remark 1 It is clear that by choosing $\mathrm{k}=0$ in the above theorem we obtain the earlier results given in [17, Thm. 5, p. 107]. Moreover, for $\mathrm{k}=1$ and $\alpha=0$ in the above theorem we get the results given in [5, Thm. 2.2]. 


\subsection{The radii of $k$-uniform convexity of order $\alpha$ of normalized $q-$ Bessel functions}

In this subsection, we shall concentrate on Jackson's second and third (or Hahn-Exton) q-Bessel functions which are defined by

$$
J_{v}^{(2)}(z ; q)=\frac{\left(q^{v+1} ; q\right)_{\infty}}{(q ; q)_{\infty}} \sum_{n \geq 0} \frac{(-1)^{n}\left(\frac{z}{2}\right)^{2 n+v}}{(q ; q)_{n}\left(q^{v+1} ; q\right)_{n}} q^{n(n+v)}
$$

and

$$
J_{v}^{(3)}(z ; q)=\frac{\left(q^{v+1} ; q\right)_{\infty}}{(q ; q)_{\infty}} \sum_{n \geq 0} \frac{(-1)^{n} z^{2 n+v}}{(q ; q)_{n}\left(q^{v+1} ; q\right)_{n}} q^{\frac{1}{2} n(n+1)}
$$

where $z \in \mathbb{C}, v>-1, \mathrm{q} \in(0,1)$ and

$$
(a ; q)_{0}=1, \quad(a ; q)_{n}=\prod_{k=1}^{n}\left(1-a q^{k-1}\right), \quad(a, q)_{\infty}=\prod_{k \geq 1}\left(1-a q^{k-1}\right) .
$$

These functions are $q$-analogue of the classical Bessel function of the first kind [23]

$$
\mathrm{J}_{v}(z)=\left(\frac{z}{2}\right)^{v} \sum_{\mathrm{k} \geq 0} \frac{(-1)^{\mathrm{k}}}{\mathrm{k} ! \Gamma(v+\mathrm{k}+1)}\left(\frac{z}{2}\right)^{2 \mathrm{k}},
$$

since

$$
\lim _{\mathrm{q} \nearrow 1} J_{v}^{(2)}((1-z) \mathrm{q} ; \mathrm{q})=\mathrm{J}_{v}(z), \quad \lim _{\mathrm{q} / 1} \mathrm{~J}_{v}^{(3)}\left(\frac{1-\mathrm{q}}{2} z ; \mathrm{q}\right)=\mathrm{J}_{v}(z)
$$

Obviously, the functions $\mathrm{J}_{v}^{(2)}(. ; \mathrm{q})$ and $\mathrm{J}_{v}^{(3)}(. ; \mathrm{q})$ do not belong to $\mathcal{A}$, and thus first we perform some natural normalization. We consider the following six normalized functions, as given by [10], originating from $J_{v}^{(2)}(. ; q)$ and $J_{v}^{(3)}(. ; q)$ : For $v>-1$,

$$
\begin{array}{ll}
f_{v}^{(2)}(z ; q)=\left(2^{v} c_{v}(q) J_{v}^{(2)}(z ; q)\right)^{\frac{1}{v}}, & f_{v}^{(3)}(z ; q)=\left(c_{v}(q) J_{v}^{(3)}(z ; q)\right)^{\frac{1}{v}},(v \neq 0) \\
g_{v}^{(2)}(z ; q)=2^{v} c_{v}(q) z^{1-v} J_{v}^{(2)}(z ; q), & g_{v}^{(3)}(z ; q)=c_{v}(q) z^{1-v} J_{v}^{(3)}(z ; q), \\
h_{v}^{(2)}(z ; q)=2^{v} c_{v}(q) z^{1-\frac{v}{2}} J_{v}^{(2)}(\sqrt{z} ; q), & h_{v}^{(3)}(z ; q)=c_{v}(q) z^{1-\frac{v}{2}} J_{v}^{(3)}(\sqrt{z} ; q),
\end{array}
$$

where $c_{v}(q)=(q ; q)_{\infty} /\left(q^{v+1} ; q\right)_{\infty}$. It is clear that each of the above functions belong to the class $\mathcal{A}$. 
In view of $[10$, Lem. 1, p.972], we know that the infinite product representations of the functions $z \mapsto \mathbf{j}_{v}^{(2)}(z ; \mathbf{q})$ and $z \mapsto \mathbf{j}_{v}^{(3)}(z ; \mathbf{q})$ are of the form

$J_{v}^{(2)}(z ; q)=\frac{z^{v}}{2^{v} c_{v}(q)} \prod_{n \geq 1}\left(1-\frac{z^{2}}{j_{v, n}^{2}(q)}\right), \quad J_{v}^{(3)}(z ; q)=\frac{z^{v}}{c_{v}(q)} \prod_{n \geq 1}\left(1-\frac{z^{2}}{l_{v, n}^{2}(q)}\right)$

where $j_{v, n}(q)$ and $l_{v, n}(q)$ denote the $n$th positive zeros of the functions $j_{v}^{(2)}(z ; q)$ and $\mathfrak{j}_{v}^{(3)}(z ; q)$, respectively.

Also, from [10, Lem. 8] we observe that the functions $z \mapsto g_{v}^{(2)}(z ; q), z \mapsto$ $\mathrm{h}_{v}^{(2)}(z ; \mathrm{q}), z \mapsto \mathrm{g}_{v}^{(3)}(z ; \mathrm{q})$ and $z \mapsto \mathrm{h}_{v}^{(3)}(z ; \mathrm{q})$ are of the form

$$
\begin{array}{ll}
\frac{\mathrm{dg}_{v}^{(2)}(z ; \mathrm{q})}{\mathrm{d} z}=\prod_{n \geq 1}\left(1-\frac{z^{2}}{\alpha_{v, n}^{2}(\mathrm{q})}\right), & \frac{\mathrm{dg}_{v}^{(3)}(z ; \mathrm{q})}{\mathrm{d} z}=\prod_{n \geq 1}\left(1-\frac{z^{2}}{\gamma_{v, n}^{2}(\mathrm{q})}\right) \\
\frac{\mathrm{dh}_{v}^{(2)}(z ; \mathrm{q})}{\mathrm{d} z}=\prod_{n \geq 1}\left(1-\frac{z}{\beta_{v, n}^{2}(\mathrm{q})}\right), & \frac{\mathrm{dh}_{v}^{(3)}(z ; \mathrm{q})}{\mathrm{d} z}=\prod_{n \geq 1}\left(1-\frac{z}{\delta_{v, n}^{2}(\mathrm{q})}\right)
\end{array}
$$

where $\alpha_{v, n}(q)$ and $\beta_{v, n}(q)$ represent the $n$th positive zeros of $z \mapsto z \cdot d J_{v}^{(2)}(z ; q) /$ $\mathrm{d} z+(1-v) J_{v}^{(2)}(z ; \mathrm{q})$ and $z \mapsto z \cdot d J_{v}^{(2)}(z ; q) / \mathrm{d} z+(2-v) J_{v}^{(2)}(z ; \mathrm{q})$, while $\gamma_{v, n}(\mathbf{q})$ and $\delta_{v, n}(q)$ are the $n$th positive zeros of $z \mapsto z \cdot d J_{v}^{(3)}(z ; q) / d z+(1-v) J_{v}^{(3)}(z ; q)$ and $z \mapsto z \cdot d J_{v}^{(3)}(z ; q) / d z+(2-v) J_{v}^{(3)}(z ; q)$.

Now, we are ready to present our results related with the radius of $k$-uniform convexity of order $\alpha$ of the normalized $q-$-Bessel functions:

Theorem 2 Let $v>-1, s \in\{2,3\}$ and $\mathrm{q} \in(0,1)$. Then, the following assertions holds true

a. Suppose that $v>0$. Then, the radius of $\mathrm{k}$-uniform convexity of order $\alpha$ of the function $z \mapsto \mathrm{f}_{v}^{(s)}(z ; q)$ is the real number $r_{k, \alpha}^{u c}\left(f_{v}^{(s)}\right)$ which is the smallest positive root of the equation

$$
1-\alpha+(1+k) r \frac{\left(f_{v}^{(s)}(r ; q)\right)^{\prime \prime}}{\left(f_{v}^{(s)}(r ; q)\right)^{\prime}}=0
$$

in $\left(0, j_{v, 1}^{\prime}(q)\right)$.

b. The radius of $\mathrm{k}$-uniform convexity of order $\alpha$ of the function $z \mapsto \mathrm{g}_{v}^{(s)}(z ; \mathrm{q})$ is the real number $\mathrm{r}_{k, \alpha}^{\mathrm{uc}}\left(\mathrm{g}_{\mathrm{v}}^{(\mathrm{s})}\right)$ which is the smallest positive root of the equation 


$$
\begin{aligned}
& ((1-v)(1+\alpha-(1+k) v)) J_{v}^{(s)}(r ; q) \\
& +(1-\alpha+2(1+k)(1-v)) r\left(J_{v}^{(s)}(r ; q)\right)^{\prime} \\
& +(1+k) r^{2}\left(J_{v}^{(s)}(r ; q)\right)^{\prime \prime}=0
\end{aligned}
$$

in $\left(0, \alpha_{v, 1}(q)\right)$.

c. The radius of $\mathrm{k}$-uniform convexity of order $\alpha$ of the function $z \mapsto \mathrm{h}_{v}^{(\mathrm{s})}(z ; \mathrm{q})$ is the real number $\mathrm{r}_{k, \alpha}^{\mathrm{uc}}\left(\mathrm{h}_{v}^{(s)}\right)$ which is the smallest positive root of the equation

$$
\begin{aligned}
& ((v-2)(v(1+k)-2(1-\alpha))) J_{v}^{(s)} \\
& \quad+((3-2 v)(1+k)+2(1-\alpha)) \sqrt{r}\left(J_{v}^{(s)}\right)^{\prime} \\
& \quad+(1+k) r\left(J_{v}^{(s)}\right)^{\prime \prime}=0
\end{aligned}
$$

in $\left(0, \beta_{v, 1}^{2}(q)\right)$, where $J_{v}^{(s)}=J_{v}^{(s)}(\sqrt{r} ; q)$.

Proof. Since the proofs for the cases $s=2$ and $s=3$ are almost the same we are going to present the proof only for the case $s=2$.

a. In $[10$, p. 979$]$ it was proven that the following equality is valid

$$
1+z \frac{\left(f_{v}^{(2)}(z ; q)\right)^{\prime \prime}}{\left(f_{v}^{(2)}(z ; q)\right)^{\prime}}=1-\left(\frac{1}{v}-1\right) \sum_{n \geq 1} \frac{2 z^{2}}{j_{v, n}^{2}(q)-z^{2}}-\sum_{n \geq 1} \frac{2 z^{2}}{j_{v, n}^{\prime 2}(q)-z^{2}}
$$

where $j_{v, n}(q)$ and $j_{v, n}^{\prime}(q)$ are the $n$th positive roots of the functions $z \mapsto J_{v}^{(2)}(z ; q)$ and $z \mapsto d J_{v}^{(2)}(z ; q) / d z$, respectively.

Now, suppose that $v \in(0,1]$. Taking into account the inequality (4), for $z \in \mathbb{D}_{j_{1}^{\prime}(q)}$ we obtain the inequality 


$$
\begin{aligned}
\operatorname{Re}\left(1+z \frac{\left(f_{v}^{(2)}(z ; q)\right)^{\prime \prime}}{\left(f_{v}^{(2)}(z ; q)\right)^{\prime}}\right) \geq 1 & -\left(\frac{1}{v}-1\right) \sum_{n \geq 1} \frac{2 r^{2}}{j_{v, n}^{2}(q)-r^{2}} \\
& -\sum_{n \geq 1} \frac{2 r^{2}}{j_{v, n}^{\prime 2}(q)-r^{2}} \\
=1 & +r \frac{\left(f_{v}^{(2)}(r ; q)\right)^{\prime \prime}}{\left(f_{v}^{(2)}(r ; q)\right)^{\prime}}
\end{aligned}
$$

where $|z|=r$. Moreover, by using triangle inequality along with the fact that $\frac{1}{v}-1>0$, we get

$$
\left|z \frac{\left(f_{v}^{(2)}(z ; q)\right)^{\prime \prime}}{\left(f_{v}^{(2)}(z ; q)\right)^{\prime}}\right| \leq-r \frac{\left(f_{v}^{(2)}(r ; q)\right)^{\prime \prime}}{\left(f_{v}^{(2)}(r ; q)\right)^{\prime}}
$$

On the other hand, observe that if we use the inequality (3), then we obtain that the above inequalities is also valid for $v>1$. Here we used tacitly that the zeros $j_{v, n}(q)$ and $j_{v, n}^{\prime}(q)$ interlace according to $[10$, Lem. $9 .$, p. 975]. The above inequalities imply for $r \in\left(0, j_{v, 1}^{\prime}(q)\right)$

$$
\inf _{|z|<r}\left[\operatorname{Re}\left(1+z \frac{\left(f_{v}^{(2)}(z ; q)\right)^{\prime \prime}}{\left(f_{v}^{(2)}(z ; q)\right)^{\prime}}\right)-k\left|z \frac{\left(f_{v}^{(2)}(z ; q)\right)^{\prime \prime}}{\left(f_{v}^{(2)}(z ; q)\right)^{\prime}}\right|-\alpha\right]=1-\alpha+(1+k) r \frac{\left(f_{v}^{(2)}(r ; q)\right)^{\prime \prime}}{\left(f_{v}^{(2)}(r ; q)\right)^{\prime}} .
$$

The function $u_{v}:\left(0, j_{v, 1}^{\prime}(q)\right) \mapsto \mathbb{R}$ defined by

$$
\begin{aligned}
u_{v}(r) & =1-\alpha+(1+k) r \frac{\left(f_{v}^{(2)}(r ; q)\right)^{\prime \prime}}{\left(f_{v}^{(2)}(r ; q)\right)^{\prime}} \\
& =1-\alpha-(1+k) \sum_{n \geq 1}\left(\frac{2 r^{2}}{j_{v, n}^{\prime 2}(q)-r^{2}}-\left(1-\frac{1}{v}\right) \frac{2 r^{2}}{j_{v, n}^{2}(q)-r^{2}}\right)
\end{aligned}
$$

is strictly decreasing since

$$
u_{v}^{\prime}(r)=-(1+k) \sum_{n \geq 1}\left(\frac{4 r j_{v, n}^{\prime 2}(q)}{\left(j_{v, n}^{\prime 2}(q)-r^{2}\right)^{2}}-\left(1-\frac{1}{v}\right) \frac{4 r j_{v, n}^{2}(q)}{\left(j_{v, n}^{2}(q)-r^{2}\right)^{2}}\right)<0
$$


for $r \in\left(0, j_{v, 1}^{\prime}(q)\right)$. Also, it can be observed that

$$
\lim _{r \searrow 0} u_{v}(r)=1-\alpha \text { and } \lim _{r / j_{v, 1}^{\prime}(q)} u_{v}(r)=-\infty .
$$

Consequently, it is obvious that the equation

$$
1-\alpha+(1+k) r \frac{\left(f_{v}^{(2)}(r ; q)\right)^{\prime \prime}}{\left(f_{v}^{(2)}(r ; q)\right)^{\prime}}=0
$$

has a unique root $r_{k, \alpha}^{u c}\left(f_{v}^{(2)}(z ; q)\right)$ in $\mathbb{D}_{\left(0, j_{v, 1}^{\prime}(q)\right)}$, where $r_{k, \alpha}^{u c}\left(f_{v}^{(2)}(z ; q)\right)$ is the radius of $k$-uniform convexity of order $\alpha$ of the function $z \mapsto$ $f_{v}^{(2)}(z ; q)$.

Taking into account Equ. (15) and (16), the rest of proof is obvious and follows by considering a similar way of concluding process as in the previous theorem. This is why we omit the rest of proof here.

Remark 2 It is obvious that by taking $\mathrm{k}=1$ and $\alpha=0$ in the above theorem we obtain the results given in [5, Thm. 2.1].

\subsection{Radius of strong starlikeness of normalized Wright and $q-$ Bessel functions}

In this subsection, our aim is to present the radius of strong starlikeness of normalized Wright and $\mathrm{q}$-Bessel functions. It is well known from [19] that a function $\mathrm{f} \in \mathcal{A}$ is said to be strong starlike of order $\gamma, 0<\gamma \leq 1$, if

$$
\left|\arg \frac{z f^{\prime}(z)}{f(z)}\right|<\frac{\pi \gamma}{2}, \quad z \in \mathbb{D}
$$

and the real number

$$
r_{\gamma}(\mathrm{f})=\sup \left\{r>0:\left|\arg \frac{z f^{\prime}(z)}{\mathrm{f}(z)}\right|<\frac{\pi \gamma}{2}, \quad \forall z \in \mathbb{D}_{\mathrm{r}}\right\}
$$

is called the radius of strong starlikeness of $f$.

The following lemma have an important place for finding our main results:

Lemma 2 [19] If $a$ is any point in $|\arg w| \leq \frac{\pi \gamma}{2}$ and if

$$
\mathrm{R}_{\mathrm{a}} \leq \operatorname{Re}[\mathrm{a}] \sin \frac{\pi \gamma}{2}-\operatorname{Im}[\mathrm{a}] \cos \frac{\pi \gamma}{2}, \quad \operatorname{Im}[\mathrm{a}] \geq 0,
$$

the disk $|w-\mathrm{a}| \leq \mathrm{R}_{\mathrm{a}}$ is contained in the sector $|\arg w| \leq \frac{\pi \gamma}{2}, 0<\gamma \leq 1$. In particular when $\operatorname{Im}[\mathrm{a}]=0$, the condition becomes $\mathrm{R}_{\mathrm{a}} \leq \mathrm{a} \sin \frac{\pi \gamma}{2}$. 
We are now in a position to present our main results related with the radii of strong starlikeness of normalized Wright and q-Bessel functions. Upcoming theorem is related with normalized Wright functions.

Theorem 3 Let $\rho>0$ and $\beta>0$. The following assertions are true:

a. The radius of strong starlikeness of $\mathrm{f}_{\rho, \beta}$ is the smallest positive root of the equation

$$
\frac{2}{\beta} \sum_{n \geq 1} \frac{r^{2}\left(\lambda_{\rho, \beta, n}^{2}+r^{2} \sin \frac{\pi \gamma}{2}\right)}{\lambda_{\rho, \beta, n}^{4}-r^{4}}-\sin \frac{\pi \gamma}{2}=0
$$

in $\left(0, \lambda_{\rho, \beta, 1}\right)$.

b. The radius of strong starlikeness of $\mathrm{g}_{\rho, \beta}$ is the smallest positive root of the equation

$$
2 \sum_{n \geq 1} \frac{r^{2}\left(\lambda_{\rho, \beta, n}^{2}+r^{2} \sin \frac{\pi \gamma}{2}\right)}{\lambda_{\rho, \beta, n}^{4}-r^{4}}-\sin \frac{\pi \gamma}{2}=0
$$

in $\left(0, \lambda_{\rho, \beta, 1}\right)$.

c. The radius of strong starlikeness of $\mathrm{h}_{\rho, \beta}$ is the smallest positive root of the equation

$$
\sum_{n \geq 1} \frac{r\left(\lambda_{\rho, \beta, n}^{2}+r \sin \frac{\pi \gamma}{2}\right)}{\lambda_{\rho, \beta, n}^{4}-r^{2}}-\sin \frac{\pi \gamma}{2}=0
$$

in $\left(0, \lambda_{\rho, \beta, 1}^{2}\right)$.

Proof. For $|z| \leq r<1,\left|z_{k}\right|=R>r$, we have from [19]

$$
\left|\frac{z}{z-z_{k}}+\frac{r^{2}}{R^{2}-r^{2}}\right| \leq \frac{R r}{R^{2}-r^{2}} .
$$

Since the series $\sum_{n \geq 1} \frac{2 r^{2}}{\lambda_{\rho, \beta, n}^{2}-r^{2}}$ and $\sum_{n \geq 1} \frac{r}{\lambda_{\rho, \beta, n}^{2}-r}$ are convergent, we arrive at

$$
\left|\frac{z f_{\rho, \beta}^{\prime}(z)}{f_{\rho, \beta}(z)}-\left(1-\frac{2}{\beta} \sum_{n \geq 1} \frac{r^{4}}{\lambda_{\rho, \beta, n}^{4}-r^{4}}\right)\right| \leq \frac{2}{\beta} \sum_{n \geq 1} \frac{\lambda_{\rho, \beta, n}^{2} r^{2}}{\lambda_{\rho, \beta, n}^{4}-r^{4}}
$$




$$
\begin{aligned}
& \left|\frac{z g_{\rho, \beta}^{\prime}(z)}{g_{\rho, \beta}(z)}-\left(1-\sum_{n \geq 1} \frac{2 r^{4}}{\lambda_{\rho, \beta, n}^{4}-r^{4}}\right)\right| \leq 2 \sum_{n \geq 1} \frac{\lambda_{\rho, \beta, n}^{2} r^{2}}{\lambda_{\rho, \beta, n}^{4}-r^{4}} \\
& \left|\frac{z h_{\rho, \beta}^{\prime}(z)}{h_{\rho, \beta}(z)}-\left(1-\sum_{n \geq 1} \frac{r^{2}}{\lambda_{\rho, \beta, n}^{4}-r^{2}}\right)\right| \leq \sum_{n \geq 1} \frac{\lambda_{\rho, \beta, n}^{2} r}{\lambda_{\rho, \beta, n}^{4}-r^{2}}
\end{aligned}
$$

for $z \in \mathbb{D}_{\lambda_{\rho, \beta, 1}}$ where $|z|=r$ and $\lambda_{\rho, \beta, n}$ stands for the $n$th positive zero of the function $\lambda_{\rho, \beta}$. Thanks to Lemma 2 , it is obvious that the disk given in (20) is contained in the sector $|\arg w| \leq \frac{\pi \gamma}{2}$, if

$$
\frac{2}{\beta} \sum_{n \geq 1} \frac{\lambda_{\rho, \beta, n}^{2} r^{2}}{\lambda_{\rho, \beta, n}^{4}-r^{4}} \leq\left(1-\frac{2}{\beta} \sum_{n \geq 1} \frac{r^{4}}{\lambda_{\rho, \beta, n}^{4}-r^{4}}\right) \sin \frac{\pi \gamma}{2}
$$

is satisfied. This inequality reduces to $\psi(r) \leq 0$ where

$$
\psi(r)=\frac{2}{\beta} \sum_{n \geq 1} \frac{r^{2}\left(\lambda_{\rho, \beta, n}^{2}+r^{2} \sin \pi \gamma / 2\right)}{\lambda_{\rho, \beta, n}^{4}-r^{4}}-\sin \frac{\pi \gamma}{2} .
$$

We note that

$$
\psi^{\prime}(r)=\frac{2}{\beta} \sum_{n \geq 1} \frac{2 r \lambda_{\rho, \beta, n}^{6}+2 r 5 \lambda_{\rho, \beta, n}^{2}+4 r^{3} \lambda_{\rho, \beta, n}^{4} \sin \pi \gamma / 2}{\left(\lambda_{\rho, \beta, n}^{4}-r^{4}\right)^{2}} \geq 0 .
$$

Moreover, $\lim _{r} \searrow 0 \psi(r)<0$ and $\lim _{r}>\lambda_{\rho, \beta, 1} \psi(r)=\infty$. Thus $\psi(r)=0$ has a unique root say $\mathcal{R}_{f_{\rho, \beta}}$ in $\left(0, \lambda_{\rho, \beta, 1}\right)$. Hence the function $f_{\rho, \beta}$ is strongly starlike in $|z|<\mathcal{R}_{\mathrm{f}_{\rho, \beta}}$.

The disk given in (21) is contained in the sector $|\arg w| \leq \frac{\pi \gamma}{2}$, if

$$
\phi(r)=2 \sum_{n \geq 1} \frac{r^{2}\left(\lambda_{\rho, \beta, n}^{2}+r^{2} \sin \pi \gamma / 2\right)}{\lambda_{\rho, \beta, n}^{4}-r^{4}}-\sin \frac{\pi \gamma}{2} \leq 0 .
$$

Also, the proof of part $(b)$ is completed by considering the limits $\lim _{\mathrm{r} \backslash \mathrm{0}} \phi(\mathrm{r})<$ 0 and $\lim _{\mathrm{r}} \lambda_{\rho, \beta, 1} \phi(\mathrm{r})=\infty$.

The proof of part $(c)$ is obvious and follows by considering the same concluding process as in the proof of part $(b)$.

Since it can be obtained desired results by repeating the same calculations in the previous theorem we present the following theorem without proof. 
Theorem 4 Let $v>-1, s \in\{2,3\}$ and $\mathbf{q} \in(0,1)$. Moreover, let $\eta_{v, n}(\mathbf{q})$ be the $\mathrm{n}$ th positive root of the function $z \mapsto \mathrm{J}_{v}^{(s)}(z ; \mathrm{q})$. Then the following assertions are true:

a. The radius of strong starlikeness of the function $\mathrm{f}_{v}^{(s)}(z ; \mathrm{q})$ is the smallest positive root of the equation

$$
\frac{2}{v} \sum_{n \geq 1} \frac{r^{2}\left(\eta_{v, n}^{2}(q)+r^{2} \sin \frac{\pi \gamma}{2}\right)}{\eta_{v, n}^{4}(q)-r^{4}}-\sin \frac{\pi \gamma}{2}=0
$$

in $\left(0, \eta_{v, 1}(\mathbf{q})\right)$, where $\eta_{v, 1}(\mathbf{q})$ is the smallest positive zero of the function $\mathrm{J}_{v}^{(s)}(z ; \mathrm{q})$.

b. The radius of strong starlikeness of $\mathbf{g}_{v}^{(s)}(z ; \mathbf{q})$ is the smallest positive root of the equation

$$
2 \sum_{n \geq 1} \frac{r^{2}\left(\eta_{v, n}^{2}(q)+r^{2} \sin \frac{\pi \gamma}{2}\right)}{\eta_{v, n}^{4}(q)-r^{4}}-\sin \frac{\pi \gamma}{2}=0
$$

in $\left(0, \eta_{v, 1}(q)\right)$.

c. The radius of strong starlikeness of $\mathrm{h}_{v}^{(s)}(z ; \mathrm{q})$ is the smallest positive root of the equation

$$
\sum_{n \geq 1} \frac{r\left(\eta_{\eta, n}^{2}(q)+r \sin \frac{\pi \gamma}{2}\right)}{\eta_{v, n}^{4}(q)-r^{2}}-\sin \frac{\pi \gamma}{2}=0
$$

in $\left(0, \eta_{v, 1}^{2}(q)\right)$.

\section{References}

[1] İ. Aktaş, Á. Baricz, Bounds for the radii of starlikeness of some q-Bessel functions, Results Math, 72 (1-2) (2017), 947-963.

[2] İ. Aktaş, Á. Baricz, H. Orhan, Bounds for the radii of starlikeness and convexity of some special functions, Turk J Math, 42 (1) (2018), 211-226.

[3] İ. Aktaş, Á. Baricz, N. Yağmur, Bounds for the radii of univalence of some special functions, Math. Inequal. Appl., 20 (3) (2017), 825-843. 
[4] İ. Aktaş, H. Orhan, Bounds for the radii of convexity of some q-Bessel functions, arXiv:1702.04549

[5] İ. Aktaş, E. Toklu, H. Orhan, Radius of Uniform Convexity of some special functions, Turk J Math, 42 (6) (2018), 3010-3024.

[6] Á. Baricz, Geometric properties of generalized Bessel functions of complex order, Mathematica, 48 (71) (2006), 13-18.

[7] Á. Baricz, Geometric properties of generalized Bessel functions, Publ. Math. Debrecen, 73 (2008), 155-178.

[8] Á. Baricz, Generalized Bessel Functions of the First Kind, Lecture Notes in Mathematics, vol. 1994, Springer-Verlag, Berlin, 2010.

[9] Á. Baricz, D.K. Dimitrov, H. Orhan, N. Yağmur, Radii of starlikeness of some special functions, Proc. Amer. Math. Soc., 144 (8) (2016), 33553367 .

[10] Á. Baricz, D.K. Dimitrov, I. Mező, Radii of starlikeness and convexity of some q-Bessel functions, J. Math. Anal. Appl., 435 (2016), 968-985.

[11] Á. Baricz, P. Kupán, R. Szász, The radius of starlikeness of normalized Bessel functions of the first kind, Proc. Amer. Math. Soc., 142 (6) (2014), 2019-2025.

[12] Á. Baricz, H. Orhan, R. Szász, The radius of $\alpha$-convexity of normalized Bessel functions of the first kind, Comput. Methods Funct. Theory, 16 (1) (2016), 93-103.

[13] Á. Baricz, S. Ponnusamy, Starlikeness and convexity of generalized Bessel functions, Integr. Transforms Spec. Funct., 21 (2010), 641-653.

[14] Á. Baricz, S. Singh, Zeros of some special entire functions, Proc. Amer. Math. Soc., 146 (5) (2018), 2207-2216.

[15] Á. Baricz, R. Szász, The radius of convexity of normalized Bessel functions of the first kind, Anal. Appl., 12 (5) (2014), 485-509.

[16] Á. Baricz, R. Szász, Close-to-convexity of some special functions, Bull. Malay. Math. Sci. Soc., 39 (1), (2016) 427-437.

[17] Á. Baricz, E. Toklu, E. Kadioğlu, Radii of starlikeness and convexity of Wright functions, Math. Commun., 23 (2018), 97-117. 
[18] Á. Baricz, N. Yağmur, Geometric properties of some Lommel and Struve functions, Ramanujan J., 42 (2) (2017), 325-346.

[19] N. Bohra, V. Ravichandran, Radii problems for normalized Bessel functions of the first kind, Comput. Methods Funct. Theory, 8 (2018), 99-123.

[20] R. K. Brown, Univalence of Bessel functions, Proc. Amer. Math. Soc., 11 (2) (1960), 278-283.

[21] E. Deniz, R. Szász, The radius of uniform convexity of Bessel functions, J. Math. Anal. Appl., 453 (1) (2017), 572-588.

[22] E. Kreyszig, J. Todd, The radius of univalence of Bessel functions, Illinois J. Math., 4 (1960), 143-149.

[23] G. N. Watson, A Treatise of the Theory of Bessel Functions, Cambridge Univ. Press, Cambridge, 1944.

[24] H. S. Wilf, The radius of univalence of certain entire functions, Illinois J. Math., (1962), 242-244.

[25] Szász R. On starlikeness of Bessel functions of the first kind. In: Proceedings of the 8th Joint Conference on Mathematics and Computer Science; 2010; Komárno, Slovakia. pp 9.

[26] E. M. Wright, On the coefficients of power series having exponential singularities.J. Lond. Math. Soc, 8 (1933), 71-79. 